\title{
Hi-Tech Bike Security using GPS and GSM
}

\author{
Sameer Mulla ${ }^{1}$, Manish Kushwaha ${ }^{1}$, Sujit Waje ${ }^{1}$, Ranjana Gupta ${ }^{1}$ \\ Computer Engineering, Indira College of Engineering and Management, Post Parandwadi, Tal-Mava, Pune ${ }^{1}$
}

\begin{abstract}
In this current world where technology is growing up day by day and scientific researchers are presenting new era of discoveries, the need for security is also increasing in all areas. At present, the vehicle usage is basic necessity for everyone. Simultaneously, protecting the vehicle against theft is also very important. The main goal of this paper is to protect the bike from any unauthorized access, using fast, easy-to-use, clear, reliable and economical fingerprint recognition technique. This bike security system intimates the status of the vehicle to the authoritative person (owner) using Global System for Mobile (GSM) communication technology. If the person is authorized, bike access is allowed. Else alert SMS will be sent to the owner and the engine will be immobilized. The prototype model for the security system is built on the embedded platform using PIC Microcontroller which controls all the processes and cost is also very stumpy. If the thief tries to steal the bike by breaking any of the security modules, the sensor which is present on the bike will sense the action of user and notify to microcontroller and then burglar alarm starts ringing. If the thief able to steal the bike in off state then by using GPS technology we can easily get the location of vehicle.
\end{abstract}

Keywords: Systems- Admin and Guest, Security and Protection- Access controls, Authentication, Verification, Unauthorized, Computer System Organization- General, User Interfaces- Graphical User Interfaces (GUI).

\section{INTRODUCTION}

In this present era the automobile thefts are increasing at a very high rate all around the world. Protecting our vehicles from such theft actions is also one challenge. Most of the anti-theft systems are developed to provide security to vehicle. One can come with a beeps, fire alarm, security camera and personal safety products.

But all these products are available commercially at a very high-price. Hence, this paper provides a tact to design and develop a low cost, trouble -free, simple vehicle theft control scheme using microcontroller. The system also includes GSM and GPS technology for communication purpose.

Below mentioned some reasons due to which vehicle protection is limited:-

1. Due to long range distance the siren cannot be heard.

2. The alarm can be disabled on theft attempts.

3. Alarm sound can be reduced in crowded areas.

In this paper existing scenarios and proposed structural design are examined, which describes the various modules and their corresponding working methods. The security system concept which we are mentioning in this paper is implemented as a prototype model.

\section{MOTIVATION}

The main objective of our work is to find a new and highly efficient bike security mechanism most relevant to user's interests. The main goal of the system is to protect our bike from getting accessed by unauthorized user and getting stolen by the thieves.

When the vehicle is stolen, no more response or alternative could be available to help the owner of the vehicle to find it back. The main goal of this paper is to protect the vehicle from any unauthorized access, using fast, easy-to-use, reliable fingerprint recognition technique.

\section{PROBLEM STATEMENT}

To develop an Hi-tech bike security mechanism that has to be enhanced and developed in such a way so that it becomes easier and smarter to catch the unauthorized user. 


\section{EXISTING SECURITY SYSTEM}

Over the few decades various Anti - Theft security system have been developed.

Radio - Frequency Identification (RFID) is used in intelligent computerized anti - theft system. RFID cards are used to provide security access. The restriction here is that keyless RFID cards can be easily stolen. In addition, key may malfunction on contact with metallic surface. Some other system use automatic mechanism which is video surveillance solution that can be fitted into the vehicle. The camera will continuously monitoring on the actions of vehicle. The main drawback of using camera system is that it will not detect actions accurately when there is change in lightening conditions around the system and inside the vehicle.

The highly developed system use Global Positioning System (GPS) to track the position of the vehicle. Global Positioning System uses global navigation satellite system. The main problem of using this GPS system is that the signal can became degraded and the receiver will not be able to provide if view of sky is limited. Weather conditions like cloud and rainfall can also affect the signals of the satellite.

\section{SYNOPSIS FOR PROPOSED SYSTEM}

The proposed system consists of following modules. Fingerprint scanner, GPS module, GSM module, Buzzer, Microcontroller, Sensor.

\subsection{Fingerprint Recognition System}

Biometric authentication is the method of identifying people by their unique characteristic. There are many types of patterns are available such as face, iris, fingerprint, retina, ear, voice, signature, hand shape. Voice, signature, hand shapes, iris images can be easily forged. Due to bad light conditioning the face lift will lead in poor accuracy.

The fingerprint biometric is one the best biometric pattern for personal detection in terms of security and reliability. This is worldwide accepted and not easy to steal or forged.

There are mainly two types of fingerprint matching. Pattern matching and minutiae matching. The Minutiae matching technique mainly based on minutiae points like location and direction of each point. The FP recognition module consists of an image capturing module, feature extraction module and pattern matching module. In minutiae matching technique the fingerprint is taken and its score is calculated and stored in template. At the time of verifying fingerprints the image of fingerprint is taken and its score is calculated. This fingerprint is also stored in template. While matching fingerprints only templates are compared, if templates are matched the access is given to user.

\subsection{Working of the System}

The security mechanism consists of two approaches: First, if the battery supply is on and system is active. When unauthorized user try to start the bike and the alert message is send on the owner's mobile phone by the system and then owner will control the ignition of bike. In second approach when battery supply is not active and bike is moved in off state then authorized user get authentication and given access using GPS and ECU which is present on microcontroller.

The main component of the system is Microcontroller. It is responsible for all monitoring and generating the inputs and outputs respectively. The user of the bike will be given three trials for his fingerprint verification; if the fingerprint is matched then the bike access is given to user. If fingerprint does not matched then system comes to conclusion that user is not authorized and alert SMS is send on the owners mobile phone.

And the camera module will take the snapshot of the person who is trying to access bike. If the wheels are rotating then user can get location of bike by sending AT commands to the system by GSM module. The owner can also control ignition of bike by sending SMS to bike engine. The embedded unit consists of ECU (Engine Control Unit) which determines the ignition timing, monitors the engine and other parameters which gathers the signals from sensor.

\subsection{GSM Module}

The GSM (Global System for Mobile Communication) acts as an intermediate between owner and system. GSM is worldwide accepted standard for digital cellular communication. GSM modems are frequently used for providing internet connectivity and many are used for sending and receiving SMS. A wireless link is provided between the owner's cell phone and MCU (Microcontroller Unit) by GSM module. PIC uses AT commands to control modems. 


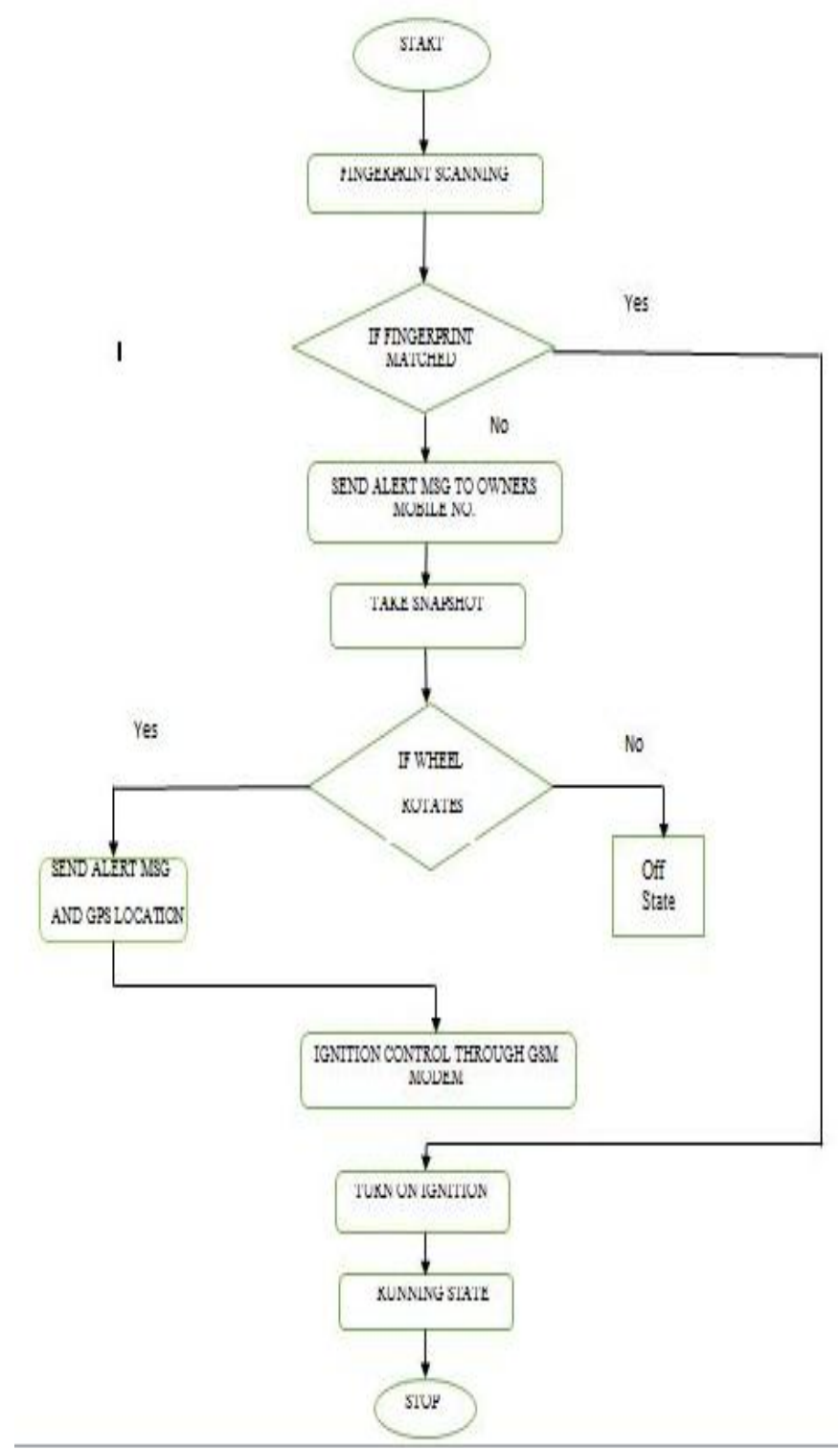

Fig. 1 Flow of the System

\section{SYSTEM DESIGN}

An anti-theft control system is consisting of following components:-

\subsection{Hardware Design}

Microcontroller

Microcontroller is acting as the BRAIN of proposed security system. The microcontroller is fed with the program containing the logic required to control the motor of the system.

Motor

The proposed system uses Dc motor. DC motor are part of the electric motors are part of electric motors using DC power as their energy source. This device transforms the electric energy into mechanical energy. The basic principle of DC motor is same as other electric motor in general, the magnetic interaction between rotor and stator that will rotate motor. The motor is connected through motor driver to the port of microcontroller.

\section{Relay}

The relay is an electromagnetic switch. When relay activates it closes the loop of ignition and the engine is started. When relay deactivated it opens the loop and engine stops. Stepper motor is connected with relay to verify operation of engine. 
Fingerprint Scanner

Fingerprint biometric is one of the most efficient, secure, cost effective technology for user authentication. It is used in the field of automobile for providing security and theft protection due to its intellectual property and commercial profit. Fingerprint scanner features

Power

Matching mode

- 3-6 volts DC

Fingerprint Enrollment

Fingerprint Matching
$-1: 1$

- 2-3 seconds

$-<1.5$ seconds

Buzzer

A buzzer is an electronic signaling device typically used in automobiles, household appliances such as microwave oven. The sensor is connected to control unit which is monitoring the actions of the intruder who is trying to steal the bike. If the intruder is trying to harm any of the component which is present on the system then the sensor will notify this to control unit and buzzer will activate.

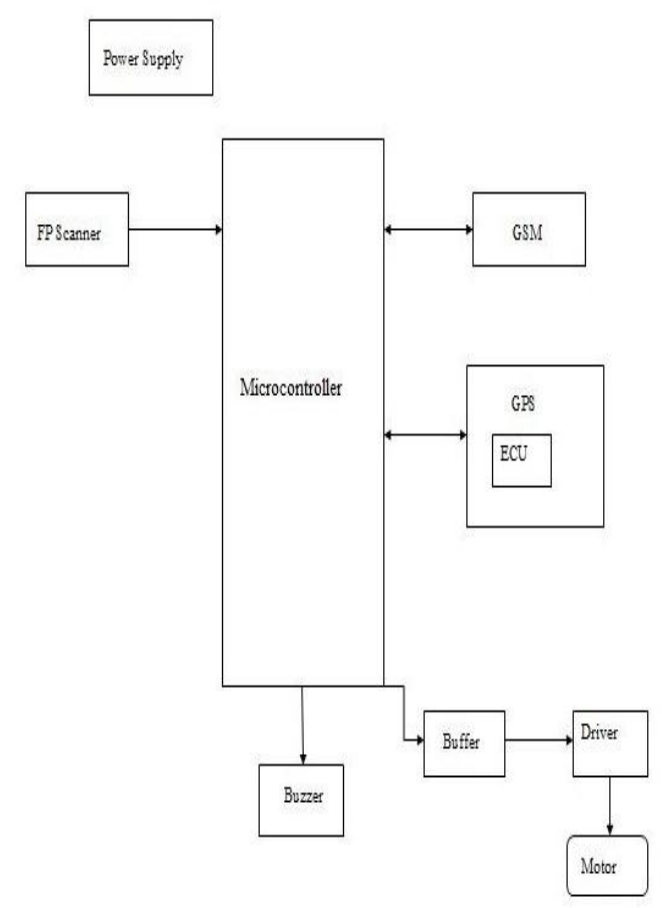

Fig. 2 Block Diagram of System

\subsection{Software Design}

\section{Android Studio IDE 2.0}

Android Studio Integrated Development Environment (IDE) is free, software application used for developing Android applications developed by Google. Android studio had replaced Eclipse IDE for developing Android applications. Android studio has a user interface that allows you to dragged and dropped different UI into place on the activity.

\section{XAMPP}

XAMPP is a free and open source cross-platform web server solution stack package developed by Apache Friends, consisting mainly of the Apache HTTP Server, MySQL database, and interpreters for scripts written in the PHP and Perl programming languages. It is a simple, lightweight Apache distribution that makes extremely easy for developers to create a local web server for testing and deployment purpose.

\section{PHP 1.5.6}

PHP is mainly server side scripting language designed primarily for web development but also used as a general purpose programming language. PHP code may be embedded into HTML or HTML5 markup, or it can be used in combination with various web template systems, web content management systems and web frameworks. PHP code is usually processed by a PHP interpreters implemented as a module in the web server or as a Common Gateway Interface (CGI) executable. The web server software combines the results of the interpreted and executed PHP code which may be any type of data, including images, with the generated web page. PHP code may also be executed with a command 
line interface (CLI) and can be used to implement standalone graphical applications. PHP is acting as a middleware between Android Studio and MySQL database.

MySQL v10.00

MySQL is an open source relational database management system (RDBMS) and widely used open-source RDBMS. All the data of register users is store in the database. All the records in the MySQL are stored in the table format.

\section{CONVENTIONAL GPS SECURITY SYSTEM}

Global Positioning System can be used to provide security to the bike in off state condition. Even it is easy to drive bike away in off state. The one case can be the bike can be totted on to the other vehicle. Thus, GPS prevents the bike lifting and theft detection. The working of GPS system is as shown in fig 3. Initially, the parking location of bike is stored. The ECU is continuously monitoring the engine function.

While bike engine is in off state but the location of bike is continuously changing, alert SMS is send on the registered user's mobile number. Hence, even when the bike is in off state the security is provided by the GPS unit. The power to the ECU is provided by internal power supply.

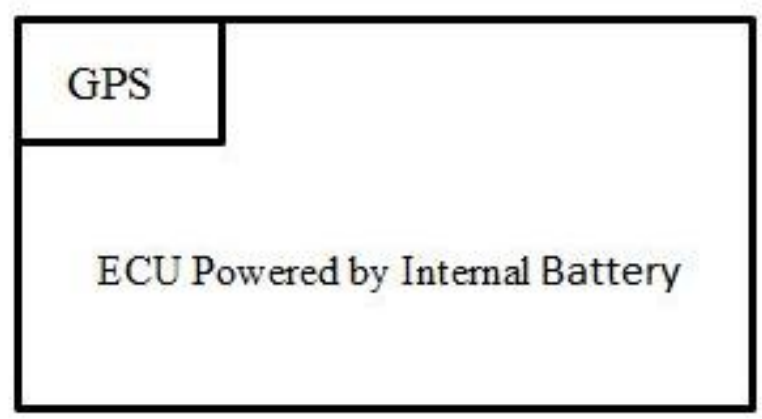

Fig. 3 GPS in security system

\section{CONCLUSION}

The aim of this paper is to improve the level of security for vehicles. As the fingerprint is a promising biometric pattern for identifying a person in terms of both security and ease of use. This is a unique method of conniving and assembling a low-cost, packed in theft control system for an automobile which is highly reliable. This is a unique method of designing and assembling a low cost, compact theft control system for automobile. This instrument is an ultimate threat to vehicle thieves. By installing this instrument it is very difficult to access by an unknown person since it is based on GSM and GPS technology. In future there is no doubt, that all of the vehicle will be embedded with this unique kit.

\section{REFERENCES}

[1] Motor theft statistics as given http://en.wikipedia.org/wiki/Motor_vehicle_theft.

[2] Sukeerti Singh and Ayushi Mhalan, Vehicle Theft Alert System using GSM, Int. Journal of Engineering Science and Technology (IJEST), May 2013.

[3] Z. M. Win and M. M. Sein, Fingerprint recognition system for low quality images, presented at the SICE Annual Conference, Waseda University, Tokyo, Japan Sept 13-18, 2011.

[4] Upendran Rajendran and Albert Joe Francis, Anti-Theft Control System Design Using Embedded System, Proc. IEEE, Vol. 85, page no. 239242, 2011.

[5] Kaisheng Zhang, Study on the Embedded Fingerprint Image Recognition System, Int. Conference of Information Science and Management Engineering, 2010.

[6] Megha Kulshrestha and V. K. Banga, Finger Print Recognition: Survey of Minutiae and Gabor Filtering Approach, Int. Journal of Computer Application(1995-8887), Volume 50No.4, July 2012.

[7] Jayanta Kumar Pany and R.N. Das Choudhury, Embedded Automobile Engine Locking System Using GSM Technology, Int. Journal of Instrumentation, Control and Automation (IJICA) ISSN : 2231-1890,Volume -1, Issue -2, 2011.

[8] Jiao She and KAisheng Zhang, Study on the Embedded Fingerprint Image Recognition System, Int. Conference of Information Science and Engineering, IEEE Computer Society, 2010.

[9] E.Walia and S.Kumar, Analysis of various biometric techniques, Int. Journal of Computer and Information Technologies, Vol.2,no.3, 2011. 